\title{
Ocular Microsporidiosis-Our Experience in a Tertiary Care Centre in North India
}

\author{
Uma Sridhar', Amil Ausaf Ur Rahman'2, Jyoti Batra1, Neelam Sapra ${ }^{3}$ \\ ${ }^{1}$ Cornea Department, ICARE Eye Hospital, Noida, India \\ ${ }^{2}$ Training Centre, ICARE Eye Hospital, Noida, India \\ ${ }^{3}$ Microbiology Depatment, Shroff Charity Eye Hospital, New Delhi, India \\ Email: u sridhar@yahoo.com
}

Received 16 June 2015; accepted 11 August 2015; published 14 August 2015

Copyright (C) 2015 by authors and Scientific Research Publishing Inc.

This work is licensed under the Creative Commons Attribution International License (CC BY).

http://creativecommons.org/licenses/by/4.0/

(c) (7) Open Access

\begin{abstract}
Microsporidia are obligate intracellular protozoal parasites. They are eukaryotic and spore forming. Increasing interest in this parasite as a pathogen in the ocular tissues in recent times is due to increasing awareness of microsporidia as an ocular pathogen and better methods of identification of the organism. It also can cause intestinal, sinus, pulmonary, muscular and renal diseases, in both immunocompetent and immunosuppressed patients. Ocular microsporidiosis can occur in isolation or as a part of systemic infections. In earlier published literature, ocular involvement in immunocompetent individuals was more in the form of stromal keratitis and immunocompromised individuals were seen to have keratoconjunctivitis. However, later studies show that this pattern has many variations. Occurrence in rainy season with exposure to muddy water and history of minor trauma is now a known factor. Identification by light microscopy from scrapings with KOH, Gram, Giemsa staining is possible. Growth of the organisms, however, is possible only by cell culture. Species identification is done by polymerase chain reaction and by electron microscopy. Immunofluorescent staining techniques are also available in advanced laboratories for species differentiation of microsporidia. Till date, treatment of ocular microsporidia has not been standardized and varies from simple debridement to use of various antibiotics, antiseptics antifungals and antiviral agents.
\end{abstract}

\section{Keywords}

Microsporidia, Keratoconjunctivitis, Stromal Keratitis

\section{Introduction}

Microsporidia are eukaryotic obligate intracellular parasitic organisms which are included as protozoans. The

How to cite this paper: Sridhar, U., Rahman, A.A.U., Batra, J. and Sapra, N. (2015) Ocular Microsporidiosis-Our Experience in a Tertiary Care Centre in North India. Open Journal of Ophthalmology, 5, 130-138.

http://dx.doi.org/10.4236/ojoph.2015.53020 
discovery of Microsporidia as pathogens in vertebrates and invertebrates was more than 100 years ago by Nageli who identified it as being causative organisms of a disease in silkworms [1]. Immunocompetent individuals can harbor microsporidia in their intestines as a part of normal flora [2]. Infection by microsporidia in humans was first reported by Matsubayashi et al. [3] in 1959. Ocular microsporidiosis was first reported by Ashton and Wirashinha in 1973 as encephalitozoan infection of cornea by Nosema sps. [4]. Earlier interest in these pathogens was due to their being opportunistic pathogens in immunocompromised patients and ocular involvement in the form of keratoconjunctivitis was also reported in these patients [5]. Recent reports in literature have been more often in immunocompetent individuals [6] [7]. The ocular manifestations include superficial punctate keratoconjunctivitis, and corneal stromal keratitis. The aim of this review is to focus on the epidemiology, pathogenesis, clinical manifestations, diagnosis and management of ocular microsporidiosis.

\subsection{Epidemiology}

Microsporidial infections occur in humans worldwide. It is identified now as a zoonotic disease as the genotypes that infect humans have been identified in animals [8]. It is also known have water borne transmission [9] and food borne transmission as a result of contaminated water used in irrigation [10]. Dairy cows' milk was also shown to be a mode of transmission of infection [11]. In areas with low socioeconomic conditions and poor sanitation, association with HIV infection is also common [12] [13]. Ocular infection occurs in immunocompetent and in immunocompromised individuals. Mode of transmission may be contact with contaminated water and soil after minor trauma. Occurrence in rainy season is common with minor epidemics being reported [14]. One of the earliest case reports of ocular involvement in humans was in a 11-year old boy who had stromal keratitis caused by microsporidia. ${ }^{4}$

\subsection{Parasitology}

Microsporidia are obligate, intracellular parasites. They have two developmental phases inside a host cell: schizogenic phase also called the feeding phase and the sporulation or sporogenic phase. The size of microsporidian spores varies from $1 \mu \mathrm{m}$ to $20 \mu \mathrm{m}$. Spores can be spherical, oval or elongate. Each spore consists of sporoplasm and a tubular, polar filament with varying number of coils, depending on the species [15].

The infection is by direct inoculation where a polar filament is discharged into the host cell and a sporoplasm is introduced. The sporoplasm multiplies inside the host cell and develops into meronts (schizonts) and sporonts. Each sporont divides to form two sporoblasts, which, in turn, develop into spores at maturation [15]. Spores may disseminate from cell to cell inside the host or can be excreted via the skin or urine. Urine to finger to eye transmission may be responsible for horizontal spread in humans [16] [17].

The only stage of microsporidia outside the host cell is that of infective spores. There are two distinct phases in the development of microsporidia: a proliferative phase (merogony) responsible for a massive increase in number inside the host cell and a sporogonic phase (sporogony), in which sporonts produce sporoblasts which mature into spores

Taxonomically, the microsporidia were grouped and classified on the basis of their natural hosts and ultrastructural features such as size of the developing and mature organisms, nucleus arrangement (monokaryon or diplokaryon), number of polar filament coils, interface with the host cell during development (e.g., direct contact with host cell cytoplasm, replication within a host-cell derived parasitophorous vacuole, replication of organisms surrounded by endoplasmic reticulum, sporogony within a parasite-generated sporophorous vesicle), and mode of cell and nuclear division (binary division, karyokinesis with delayed cytokinesis) [15].

\subsection{Genus and Species Specific Characteristics}

There are seven genera that infect humans. These parasites can be distinguished by their developmental cycle and host-parasite relationships in infected cells.

\subsubsection{Encephalitozoon}

Encephalitozoon cuniculi are unikaryotic. They develop within host cells in vacuoles bounded by a membrane that is thought to be of host cell origin. The spores are ellipsoid and measure approximately $2.5 \times 1.5 \mathrm{~mm}$ They have a corrugated exospore surface, a thick endospore, four to seven (usually five or six) coils of the polar tubules, and often a polar vacuole [1] [18]. 


\subsubsection{Nosema}

Some of the structures lie in cysts bounded by membranes, but most of the parasites are in direct contact with the cytoplasm. There is no vacuole present. Spores are oval, measuring approximately $2.5-5 \mathrm{~mm} \times 2 \mathrm{~mm}$. Nuclei in the diplokaryon arrangement are seen in all forms. There is diplosporoblastic sporogony. The polar tube has about 11 coils [1] [18].

\subsubsection{Pleistophora}

Organisms resembling microsporidia of the genus Pleistophora have been recognized in one human patient. The spores were oval, approximately $2.8 \times 3.2-3.4 \mathrm{~mm}$. Not all developmental stages were seen. Unkaryotic with sporophorous vesicle. The spore wall is typical for many microsporidia, having a thin electron-dense exospore layer, a thick electron-lucent endospore, and a thin internal plasmalemma. Approximately 11 cross sections of the polar tubule were recognized in spores [1] [18].

\subsubsection{Vittaform Cornea}

Spores measure $3.05-4.55 \times 0.77-1.27 \mathrm{~mm}$. It is diplokaryotic. There is no vacuole in direct contact with host cell cytoplasm. Band like sporonts are seen. All stages are surrounded by a cistern of host endoplasmic reticulum [1] [18].

\subsubsection{Enterocytozoon}

Enterocytozoon bienusi is the microsporidian parasite most commonly recognized in humans and, so far, has been detected only in enterocytes. All stages are seen in direct contact with the host cell cytoplasm. Early developmental stages may have diplokaryotic nuclei but later stages display isolated nuclei. The parasites are small, approximately 2 to $4 \mathrm{~mm}$ in diameter, and have a simple plasma membrane during the early stages of division. Spores are approximately $1.1-1.6 \times 0.7-1.0 \mathrm{~mm}$ in tissue section and differ from other microsporidia by having a very thin endospore layer [1] [18].

The other two genera are Trachipleistophora and Anncaliia (Brachiola) [18]. Classification of microsporidia in humans has depended on transmission electron microscopy (TEM) [19]. The presence of a polar tubule classifies an organism as a member of the phylum Microspora. Species differentiation using ultrastructural examinations is usually possible.

The fine structure of the spores with the unique coiled polar tube, the nature of host-parasite interface, and the method of division are criteria for diagnosis and species differentiation of microsporidia [18].

\subsection{Non Ocular Presentation}

Microsporidia can cause a variety of human diseases, involving multiple organ systems which include intestinal, ocular, sinus, pulmonary, muscular and renal diseases, in both immunocompetent as well as immunocompromised patients [20]. A case report of a Japanese boy with severe headache, vomiting and seizures in whom microsporidia was isolated by inoculation of mouse with his cerebrospinal fluid and urine was published in 1959 [21]. After this case report, many years ellapsed before a similar case was reported [18].

E. bieneusi and E. intestinalis are common pathogens in HIV infected patients with severe immunodeficiency and low CD 4 counts [22]. Enterocytozoon bieneusi and Encephalitozoon intestinalis have also been associated with cholangitis including sclerosing cholangitis [23]. Hepatitis and peritonitis may also be caused by microsporidia [18].

\subsection{Ocular Manifestations}

Keratitis in a Sri Lankan boy was reported in 1973 [4] by Ashton and Wirashinha in the British Journal of Ophthalmology which was histopathologically proven as microsporidiosis. Initial reports of microsporidiosis were in HIV positive patients. Cases of related cases of keratoconjunctivitis typically described the lesions as coarse, punctate epithelial lesions which may stain positive with fluorescein dye. Organism found in conjunctiva and cornea was Encephalitozoon hellem [24] [25]. In a case series from New York, keratoconjunctivitis in HIV positive individuals revealed Encephalitozoon cuniculi spores on conjunctival biopsy [26], In HIV positive patients, chronic keratoconjunctivitis has been more commonly found, whereas both deep stromal keratitis and keratoconjunctivitis have been described in immunocompetent individuals [27]-[29]. Contact lenses may harbor 
the organisms and cause corneal infections. In a case series, of microsporidial keratitis reported from Singapore, 20.1\% (25 out of 124) had a history of contact lens wear [30].

Domestic animals such as cats may harbor the organism and close contact with them may cause ocular infection [24]. Use of topical corticosteroids can also predispose to superadded infection with microsporidia. Infection after corneal grafting has been reported due to local immunosuppression with corticosteroids [31].

Exposure to muddy water, especially in rainy season has been reported as a predisposing factor in causing an outbreak in Singapore [32].

\subsection{Spectrum of Ocular Microsporidiosis}

Deep stromal keratitis and superficial punctate keratopathy are the main manifestations in the cornea. Conjunctival involvement may occur along with the keratitis. Keratoconjunctivits mimicking adenoviral keratoconjunctivitis may occur especially in the rainy season. A mixed follicular and papillary reaction may occur in the conjunctiva. Sclera and uvea may also be involved.

In cases of keratoconjunctivitis, the presenting symptoms are photophobia, blurred vision, and foreign body sensation. Conjunctiva usually is chemosed, with decreased luster. The corneal involvement is in the form of superficial punctate keratopathy with coarse fluorescein staining and non-staining epithelial opacities. The lesions are limited to the level of the epithelium and can be debrided.

Several case series describing keratoconjunctivits in immunosuppressed and in later publications, immunocompetent patients have been published in the last two decades [26]-[32].

\subsection{Our Experience of Keratoconjunctivitis}

Between August 2013 to March 201425 cases of microsporidiosis were seen in our opd. All patients had history of exposure to rain water or to muddy water. A family of seven patients had exposure to water from their overhead tank and presented with a similar picture of conjunctival congestion, chemosis raised epithelial lesions and some target shaped epithelial lesions (Figure 1 and Figure 2). Corneal scraping in all the patients was done and stained with Gram stain.

Spores of microsporidia were detected in all the specimens.

Most of the patients who were clinically suspected to have microsporidial keratitis in our opd were subjected to corneal scraping. All the specimens were either stained with Gram stain or with Geimsa stain (Figure 3). All the specimens were showed spores of microsporidia.

Patient responded to topical fluoroquinolones and oral albendazole $400 \mathrm{mg}$ once daily for seven days.

Three patients also showed signs of anterior uveitis on follow up with nummular corneal opacities and had to

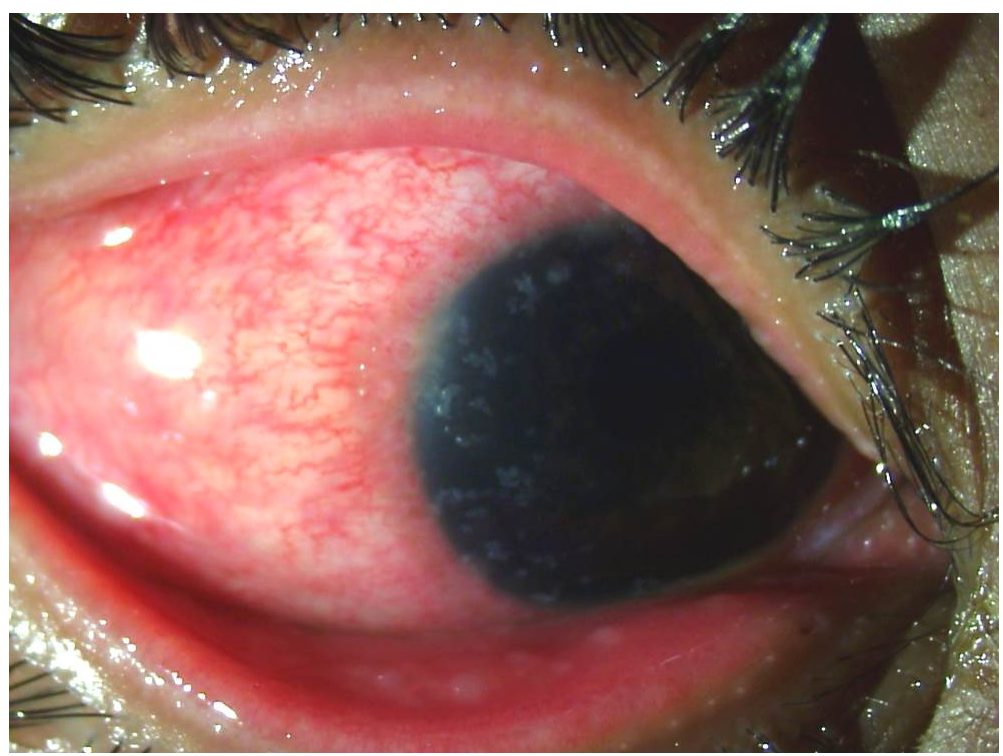

Figure 1. Raised coarse epithelial lesions on cornea in a case of microsporidia keratoconjunctivitis. 


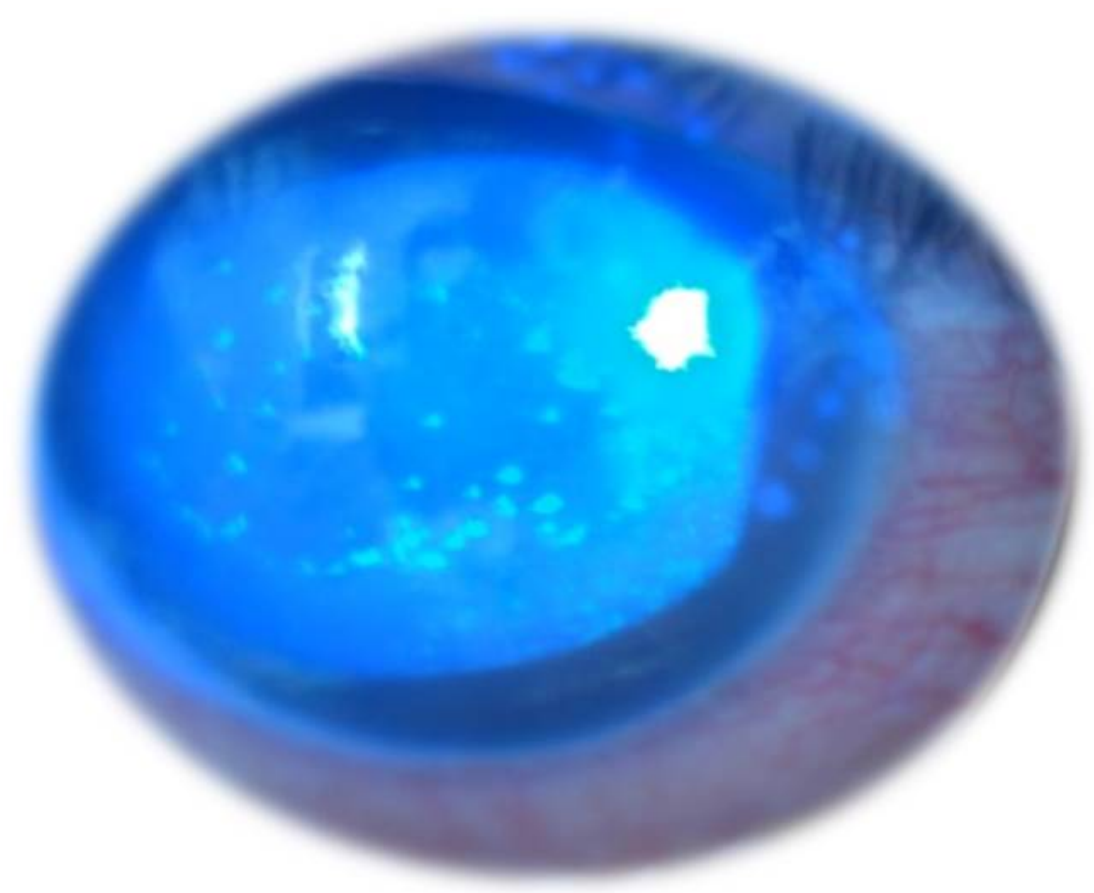

Figure 2. Fluorescein staining of punctate raised epithelial lesions in acase of microsporidia keratoconjunctivitis.

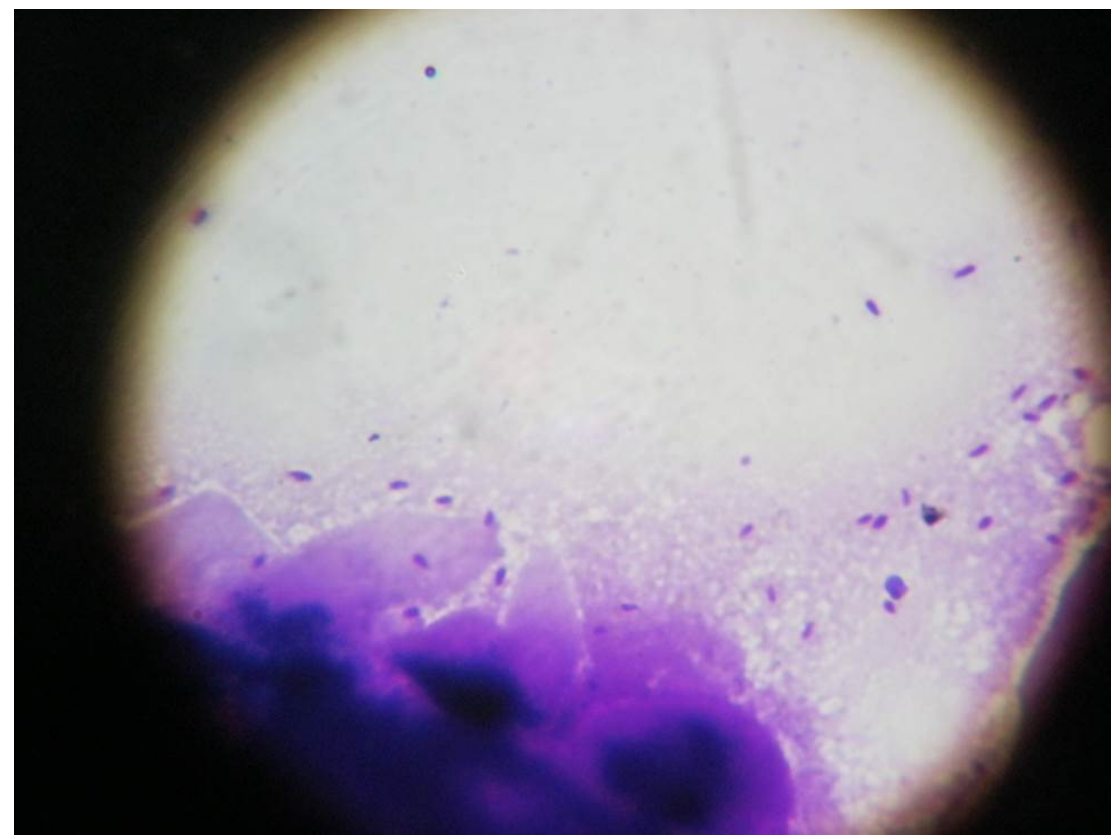

Figure 3. Giemsa staining showing microsporidia spores.

be given a short course of topical steroids.

\section{Stromal Keratitis}

Stromal keratitis due to microsporidia is rarer than superficial keratoconjunctivitis. It occurs mainly in immunocompetent patients. Stromal keratitis due to microsporidia can be mistaken for Herpes Simplex virus keratitis. A case report of a patient who had dense greyish white stromal infiltrates in the cornea which had a crystalline keratopathy-like pattern and was treated initially as Herpes simplex virus stromal keratitis was reported by Font 
et al. [33]. Corneal biopsy showed microsporidial keratitis. Since there was no improvement with oral albendazole and topical fumagillin even after 6 weeks, lamellar keratoplasty and later penetrating keratoplasty (PK) was done. The organism was identified as Nosema corneum (renamed Vittaforma corneae).

In our experience, a 70 year old gentleman presented with a diagnosis of chronic endophthalmitis in his right eye, having undergone cataract surgery and trabeculectomy elsewhere. He had perception of light with inaccurate projection in that eye. Cornea showed $2 \times 3 \mathrm{~mm}$ area of crystalline infiltrates in the stroma near the temporal cataract surgery wound, (Figure 4). Corneal scraping did not reveal any organisms. He was empirically treated with fortified cefazoline and fortified amikacin, suspecting atypical mycobacterial infection. Vitritis was seen on ultrasound B scan and he was given intravitreal injections of ceftazidime. Vitreous biopsy did not show any organism. Topical and systemic steroids were added to the treatment regimen as the patient developed more inflammation in the posterior segment and the eye was now painful. Decision was made to eviscerate the right eye as the corneal and posterior segment condition worsened with no visual prognosis. Corneal button on histopathology showed plenty of microsporidial spores (Figure 5). The species identification was not done. This was

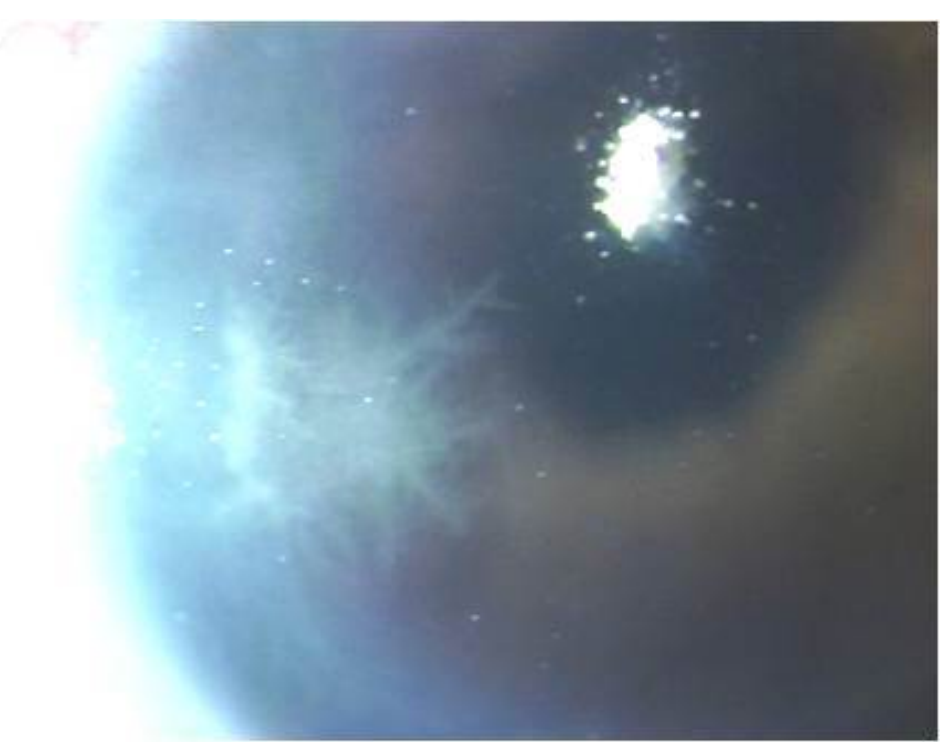

Figure 4. Infectious crystalline keratopathy due to microsporidia.

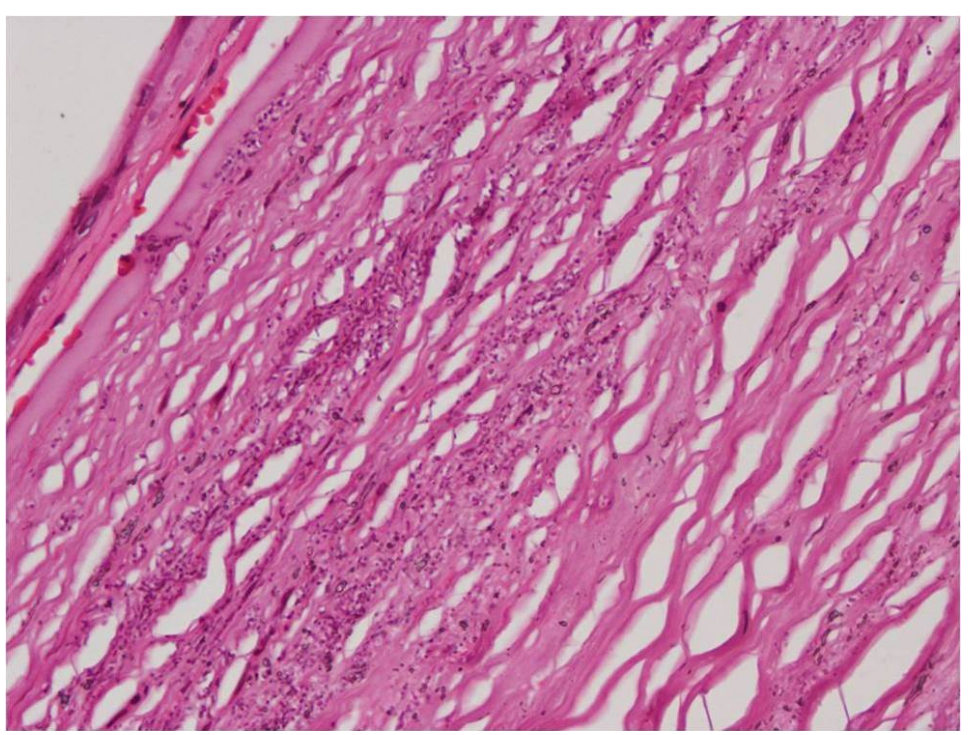

Figure 5. Histopathology of corneal button of the case of infectious crytalline keratopathy due to microsporidia. 
a case of crystalline keratopathy due to microsporidia which was not clinically diagnosed and was negative on scraping. Associated posterior segment inflammation was also a feature.

Another case report of stromal keratitis mistakenly diagnosed as viral keratitis in an 82-year-old patient was reported by Fogla et al. [34]. Initially the peripheral mid stromal infiltrates seemingly resolved with acyclovir and topical prednisolone acetate. The lesion recurred a month later as a full thickness stromal infiltrate. therapeutic penetrating keratoplasty was performed and the histopathology of the corneal button showed microsporidia spores.

Cellular reaction in the anterior chamber is common but posterior segment involvement from microsporidial infection rare. Meitz et al. [35] have reported a case of sclerouveitis with retinal detachment. There was no corneal infiltrate in the sixty six year old woman who had progressive loss of vision. There were keratic precipitates in the cornea. Diagnostic vitrectomy revealed infection with microsporidia; she was treated with oral albendazole $400 \mathrm{mg}$ twice daily. Endophthalmitis due to microsporidia was also reported in a patient with acute myelogenous leukemia [36].

\section{Diagnostic Methods}

\subsection{Non Invasive Techniques}

Clinically diagnosis of microsporidia has been done in cases of keratoconjunctivitis based on history of exposure to muddy water especially in the rainy season and the presence of coarse epithelial lesions along with conjunctival chemosis and follicular and papillary reaction. Stromal keratitis however may be more difficult to distinguish from Herpes simplex stromal keratitis clinically. When the infiltrate does not respond to topical antivirals and steroids, then resort has to be made to other means like confocal microscopy and corneal biopsy. Sometimes therapeutic penetrating or lamellar keratoplasty and histopathology may be the only means of diagnosis.

\subsection{Confocal Microscopy}

Hyperreflective spots seen on confocal microscopy in keratoconjunctivitis due to microsporidia have been reported and the diagnosis later confirmed by corneal scraping [37]. In stromal keratitis also confocal microscopy has been useful to detect microsporidia and the diagnosis has been later confirmed by biopsy [38]. Confocal microscopy, thus may be a simple and non-invasive technique to detect the organism in deep seated infections and to monitor the effectiveness of treatment.

\subsection{Microbiological Diagnosis}

Corneal and conjunctival scraping can be effective in diagnosis. Various staining methods have been used. 10\% $\mathrm{KOH}$ mount (potassium hydroxide) plain or with calcofluor white.

\section{Laboratory Diagnosis}

Identification by light microscopy from scrapings with $\mathrm{KOH}, \mathrm{KOH}+\mathrm{CFW}$ (potassium hydroxide plus calcofluor white), Gram, Giemsa and modified Ziehl-Neelsen (1\% $\mathrm{H}_{2} \mathrm{SO}_{4}$ cold) staining is possible. $\mathrm{KOH}+\mathrm{CFW}$ staining is observed under a fluorescence microscope with cube $\mathrm{U}$ having filter combinations for the excitation spectrum region near $365 \mathrm{~nm}$ for a DAPI (4', 6'-diamidino-2-phenylindole) stain. In various studies, the $\mathrm{KOH}+\mathrm{CFW}$ stain along with the modified Ziehl-Neelsen stain most frequently detected microsporidia, followed by the Gram stain, while Giemsa staining had the least detection efficacy among the four. $\mathrm{KOH}+\mathrm{CFW}$ and acid-fast stains were to be most efficient (29/30 [96.7\%] and 28/30 [93.3\%], respectively) in the diagnosis of microsporidial keratitis.

Microsporidal spores as observed under various stains on corneal scrapings. a) KOH + CFW stain (magnification, $\times 1000$ ). Organisms were seen as bright turquoise to white oval bodies, often clustered in groups, against a relatively dark background. The spores displayed variable fluorescence intensities. Depending on the orientation of the microsporidia, the anterior end appeared concave. b) Gram stain (magnification, $\times 1000$ ). Spores appeared ovoid and refractile and bright purple, resembling gram-positive organisms. The spores were scattered or highly clustered within the cytoplasm of occasional epithelial cells. Microsporidial spores show a dark staining belt girding them either diagonally or equatorially. c) Giemsa stain (magnification, $\times 1000$ ). This stain is not taken up by the cell wall, and only the cytoplasm gets stained. The spores appear smaller than those in the other stains. 
There was also poor differentiation from other bacteria and debris. The darkly stained belt could be identified in 18/30 cases, aiding preliminary diagnosis. d) Modified Ziehl-Neelsen stain (magnification, $\times 1000$ ). Except for two, all cases of microsporidial spores were acid fast $\left(1 \% \mathrm{H}_{2} \mathrm{SO}_{4}\right)$. The acid-fast spores appeared bright red on a blue background, and a posterior vacuole and central diagonal strip within the spores were often visible. Bacteria and other cell debris appeared blue, owing to methylene blue counterstain.

Growth of the organisms, however, is possible only by cell culture. Species identification is done by polymerase chain reaction and by electron microscopy. Immunofluorescent staining techniques are also available in advanced laboratories for species differentiation of microsporidia.

\section{References}

[1] Nageli, K.W. (1857) Uber die neue Krankheit der Seidenraupe und verwandte Organismen. Bot Z, 15, 760-761.

[2] Nkinin, S.W., Asonganyi, T., Didier, E.S., et al. (2007) Microsporidian Infection is Prevalent in Healthy People in Cameroon. Journal Clinical Microbiology, 45, 2841-2846. http://dx.doi.org/10.1128/JCM.00328-07

[3] Matsubayashi, H., Koike, T., Mikata, T. and Hagiwara, S. (1959) A Case of Encephatitozoon like Body Infection in Man. Archives of Pathology, 67, 181-187.

[4] Ashton, N. and Wirashinha, P.A. (1973) Encephalitozoonosis (Nosematosis) of the Cornea. British Journal of Ophthalmology, 57, 669-674. http://dx.doi.org/10.1136/bjo.57.9.669

[5] Cali, A., Meisler, D.M., Lowder, C.Y., et al. (1991) Corneal Microsporidiosis: Characterization and Identification. The Journal of Protozoology, 38, S215-S217.

[6] Chan, C.M., Theng, J.T., Li, L., et al. (2003) Microsporidial Keratoconjunctivitis in Healthy Individuals: A Case Series. Ophthalmology, 110, 1420-1425. http://dx.doi.org/10.1016/S0161-6420(03)00448-2

[7] Quek, D.T., Pan, J.C., Unny Krishnan, P. and Zhao, P.S. (2011) Teoh SCB Microsporidial Keratoconjunctivitis in the Tropics: A Case Series. The Open Ophthalmology Journal, 5, 42-47. http://dx.doi.org/10.2174/1874364101105010042

[8] Mathis, A., Weber, R. and Deplazes, P. (2005) Zoonotic Potential of the Microsporidia. Clinical Microbiology Reviews, 18, 423-445. http://dx.doi.org/10.1128/CMR.18.3.423-445.2005

[9] Didier, E.S., Stovall, M.E., Green, L.C., et al. (2004) Epidemiology of Microsporidiosis: Sources and Modes of Transmission. Veterinary Parasitology, 126, 145-166.

[10] Calvo, M., Carazo, M., Arias, M.L., et al. (2004) Prevalence of Cyclospora sp., Cryptosporidium sp., Microsporidia and Fecal coliform Determination in Fresh Fruit and Vegetables Consumed in Costa Rica. Archivos Latinoamericanos de Nutrición, 54, 428-432.

[11] Lee, J.H. (2008) Molecular Detection of Enterocytozoon bieneusi and Identification of a Potentially Human-Pathogenic Genotype in Milk. Applied and Environmental Microbiology, 74, 1664-1666. http://dx.doi.org/10.1128/AEM.02110-07

[12] Sarfati, C., Bourgeois, A., Menotti, J., Liegeois, F., Moyou-Somo, R., Delaporte, E., et al. (2006) Prevalence of Intestinal Parasites Including Microsporidia in Human Immunodeficiency Virus-Infected Adults in Cameroon: A CrossSectional Study. The American Journal of Tropical Medicine and Hygiene, 74, 162-164.

[13] Chacin-Bonilla, L., Panunzio, A.P., Monsalve-Castillo, F.M., Parra-Cepeda, I.E. and Martinez, R. (2006) Microsporidiosis in Venezuela: Prevalence of Intestinal Microsporidiosis and Its Contribution to Diarrhea in a Group of Human Immunodeficiency Virus-Infected Patients from Zulia State. The American Journal of Tropical Medicine and Hygiene, 74, 482-486.

[14] Tan, J., Lee, P., Lai, Y., Hishamuddin, P., Tay, J., Tan, A.L., Chan, K.S., Lin, R., Tan, D., Cutter, J. and Goh, K.T. (2013) Microsporidial Keratoconjunctivitis after Rugby Tournament, Singapore. Emerging Infectious Diseases, 19, 1484-1486. www.cdc.gov/eid

[15] Current, W.L. and Owen, R.L. (1989) Cryptosporidiosis and Microsporidiosis. In: Farthing, M.J.G. and Keusch, G.T., Eds., Enteric Infection. Mechanisms, Manifestations and Management, 11th Edition, Chapman and Hall, London, 203207.

[16] Bryan, R.T., Cali, A., Owen, R.L. and Spencer, H.C. (1991) Microsporidia: Opportunistic Pathogens in Patients with AIDS. In: Sun, T., Ed., Progress in Clinical Parasitology, Vol. 2, Field and Wood Medical Publishers, New York, 126.

[17] Lowder, C.Y. (1993) Ocular Microsporidiosis. International Ophthalmology Clinics, 33, 145-151. http://dx.doi.org/10.1097/00004397-199303310-00012

[18] Sharma, S., Das, S., Joseph, J., Vemuganti, G.K. and Murthy, S. (2011) Microsporidial Keratitis: Need for Increased Awareness. Survey of Ophthalmology, 56, 1-22.

[19] Sprague, V. and Va'vra, J. (1977) Systematics of the Microsporidia. In: Bulla Jr., L.A. and Cheng, T.C., Eds., Com- 
parative Pathobiology, Vol. 2. Plenum Press, New York, 1-510.

[20] Weber, R., Bryan, R.T., Schwartz, D.A. and Owen, R.L. (1994) Human Microsporidial Infections. Clinical Microbiology Reviews, 7, 426-461.

[21] Franzen, C. (2008) Microsporidia: A Review of 150 Years of Research. The Open Parasitology Journal, 2, 1-34. http://dx.doi.org/10.2174/1874421400802010001

[22] Kotler, D.P. and Orenstein, J.M. (1998) Clinical Syndromes Associated with Microsporidiosis. Advances in Parasitology, 40, 321-349. http://dx.doi.org/10.1016/S0065-308X(08)60126-8

[23] Costa, S.F. and Weiss, L.M. (2000) Drug Treatment of Microsporidiosis. Drug Resistance Updates, 3, 384-399. http://dx.doi.org/10.1054/drup.2000.0174

[24] Didier, E.S., Didier, P.J., Friedberg, S.M., Stenson, D.N., Orenstein, J.M., Vee, R.W., et al. (1991) Isolation and Characterization of a New Human Microsporidian, Encephalitozoon hellem (n. sp.), from Three AIDS Patients with Keratoconjunctivitis. The Journal of Infectious Diseases, 163, 617-621. http://dx.doi.org/10.1093/infdis/163.3.617

[25] Grossnikiaus, H.E., Diesenhouse, M.C., Wilson, L.A., Corrent, G.F., Visvesvara, G.S. and Bryan, R.T. (1993) Treatment of Microsporidial Keratoconjunctivitis with Topical Fumagillin. American Journal of Ophthalmology, 115, $293-$ 298. http://dx.doi.org/10.1016/S0002-9394(14)73578-0

[26] Friedberg, D.N., Stenson, S.M., Orenstein, J.M., Tierno, P.M. and Charles, N.C. (1990) Microsporidial Keratoconjunctivitis in Acquired Immunodeficiency Syndrome. Archives of Ophthalmology, 108, 504-508. http://dx.doi.org/10.1001/archopht.1990.01070060052047

[27] Joseph, J., Sridhar, M.S., Murthy, S. and Sharma, S. (2006) Clinical and Microbiological Profile of Microsporidial Keratoconjunctivitis in Southern India. Ophthalmology, 113, 531-537. http://dx.doi.org/10.1016/j.ophtha.2005.10.062

[28] Moon, S.J., Mann, P.M. and Matoba, A.Y. (2003) Microsporidial Keratoconjunctivitis in a Healthy Patient with a History of LASIK Surgery. Cornea, 22, 271-272. http://dx.doi.org/10.1097/00003226-200304000-00020

[29] Sridhar, M.S. and Sharma, S. (2003) Microsporidial Keratoconjunctivitis in a HIV-Seronegative Patient Treated with Debridement and Oral Itraconazole. American Journal of Ophthalmology, 136, 745-746. http://dx.doi.org/10.1016/S0002-9394(03)00391-X

[30] Loh, R.S., Chan, C.M., Ti, S.E., Lim, L., Chan, K.S. and Tan, D.T.H. (2009) Emerging Prevalence of Microsporidial Keratitis in Singapore: Epidemiology, Clinical Features, and Management. Ophthalmology, 116, 2348-2353. http://dx.doi.org/10.1016/j.ophtha.2009.05.004

[31] Kakrania, R., Joseph, J., Vaddavalli, P.K., Gangopadhyay, N. and Sharma, S. (2005) Microsporidia Keratoconjunctivitis in a Corneal Graft. Eye, 20, 1314-1315. http://dx.doi.org/10.1038/sj.eye.6702178

[32] Das, S., Sharma, S., Sahu, S.K., Nayak, S.S. and Kar, S. (2008) New Antimicrobial Spectrum of Epidemic Keratoconjunctivitis: Clinical and Laboratory Aspects of an Outbreak. British Journal of Ophthalmology, 92, 861-862.

[33] Font, R.L., Samaha, A.N., Keener, M.J., Chevez-Barrios, P. and Goosey, J.D. (2000) Corneal Microsporidiosis. Report of Case, Including Electron Microscopic Observations. Ophthalmology, 107, 1769-1775. http://dx.doi.org/10.1016/S0161-6420(00)00285-2

[34] Fogla, R., Padmanabhan, P., Therese, K.L., Biswas, J. and Madhavan, H.N. (2005) Chronic Microsporidial Stromal Keratitis in an Immunocompetent, Noncontact Lens Wearer. Indian Journal of Ophthalmology, 53, 123-125. http://dx.doi.org/10.4103/0301-4738.16177

[35] Mietz, H., Franzen, C., Hoppe, T. and Bartz-Schmidt, K.U. (2002) Microsporidia-Induced Sclerouveitis with Retinal Detachment. Archives of Ophthalmology, 120, 864-865.

[36] Yoken, J., Forbes, B., Maguire, A.M., Prenner, J.L. and Carpentieri, D. (2002) Microsporidial Endophthalmitis in a Patient with Acute Myelogenous Leukemia. Retina, 22, 123-125. http://dx.doi.org/10.1097/00006982-200202000-00028

[37] Shah, G.K., Pfister, D., Probst, L.E., Ferrieri, P. and Holland, E. (1996) Diagnosis of Microsporidial Keratitis by Confocal Microscopy and the Chromatrope Stain. American Journal of Ophthalmology, 121, 89-91. http://dx.doi.org/10.1016/S0002-9394(14)70538-0

[38] Sagoo, M.S., Mehta, J.S., Hau, S., Irion, L.D., Curry, A. and Bonshek, R.E. (2007) Microsporidium Stromal Keratitis: In Vivo Confocal Findings. Cornea, 26, 870-873. http://dx.doi.org/10.1097/ICO.0b013e31806c7a3c 